\title{
Stent-graft surface movement after endovascular aneurysm repair: baseline parameters for prediction, and association with migration and stent-graft-related endoleaks
}

\author{
Ulrika Asenbaum ${ }^{1} \cdot$ Maria Schoder $^{1} \cdot$ Ernst Schwartz ${ }^{2} \cdot$ Georg Langs $^{2} \cdot$ Pascal Baltzer $^{1} \cdot$ Florian Wolf $^{1}$. \\ Alexander M. Prusa ${ }^{3} \cdot$ Christian Loewe $^{1} \cdot$ Richard Nolz $^{1}$ (ID
}

Received: 21 January 2019 /Revised: 25 April 2019 / Accepted: 22 May 2019 /Published online: 27 June 2019

(C) The Author(s) 2019

\begin{abstract}
Objectives To evaluate the influence of baseline parameters on the occurrence of stent-graft surface movement after endovascular aneurysm repair (EVAR) and to investigate its association with migration and stent-graft-related endoleaks (srEL).

Methods In this retrospective, cross-sectional study, three-dimensional surface models of the stent-graft, delimited by landmarks using custom-built software, were derived from the pre-discharge and last follow-up computed tomography angiography (CTA). Stent-graft surface movement in the proximal anchoring zone between these examinations was considered significant at a threshold of $9 \mathrm{~mm}$. The Cox proportional hazards model was used to determine baseline variables associated with the occurrence of stent-graft surface movement. The association between migration and srEL with stent-graft surface movement was tested with the chi-square and the Fisher exact test, respectively.

Results Stent-graft surface movement was observed in 54 (28.9\%) of 187 patients. Multivariate analysis revealed that age ([HR] 1.05; $p=0.017)$, proximal neck diameter ([HR] 5.07; $p<0.001)$, infrarenal aortic neck angulation $([\mathrm{HR}] 1.02, p=0.002)$, and proximal neck length ([HR] $0.62, p<0.001)$ were significantly associated with the occurrence of stent-graft surface movement. Migration and srEL occurred in $17(31.5 \%)$ and $5(9.3 \%)$ patients, with and $11(8.3 \%)$ and $2(1.5 \%)$ without stent-graft surface movement $(p<0.001, p=0.022)$.

Conclusions Age, neck diameter, infrarenal neck angulation, and proximal neck length were significantly associated with the occurrence of stent-graft surface movement. Apart from possible use of adjunctive sealing systems, concerned patients may benefit from regular CTA surveillance, enabling timely diagnosis of subtle changes of stent-graft position.

Key Points

- Stent-graft surface movement, demonstrating subtle, three-dimensional changes in stent-graft position in the proximal anchoring zone, can be derived from CTA examinations.

- Age, proximal neck diameter, and infrarenal neck angulation were significantly associated with an increased incidence of stentgraft surface movement. Stent-graft surface movement is significantly more frequent in patients with stent-graft migration and stent-graft-related endoleaks.

- Consideration of risk factors for stent-graft surface movement may help to identify patients who might benefit from regular CTA surveillance and timely diagnosis of subtle changes of stent-graft position, enabling re-interventions to prevent migration and srEL.
\end{abstract}

Electronic supplementary material The online version of this article (https://doi.org/10.1007/s00330-019-06282-w) contains supplementary material, which is available to authorized users.

Richard Nolz

richard.nolz@meduniwien.ac.at

1 Division of Cardiovascular and Interventional Radiology,

Department of Bio-medical Imaging and Image-Guided Therapy,

Medical University of Vienna - Vienna General Hospital, Waehringer

Guertel 18-20, A-1090 Vienna, Austria
2 Computational and Imaging Research Laboratory, Department of Bio-medical Imaging and Image-Guided Therapy, Medical University of Vienna, Vienna, Austria

3 Department of Surgery, Medical University of Vienna, Vienna, Austria 
Keywords Aortic aneurysm $\cdot$ Abdominal aorta $\cdot$ Stent-graft $\cdot$ Computed tomography angiography $\cdot$ Movement risk factors

\author{
Abbreviations \\ AAA Abdominal aortic aneurysm \\ BMI Body mass index \\ CTA Computed tomography angiography \\ EVAR Endovascular aneurysm repair \\ IFU Instructions for use \\ PMA Psoas muscle area \\ RSD Renal artery to stent-graft distance \\ srEL Stent-graft-related endoleak \\ T2EL Type 2 endoleak
}

\section{Introduction}

The robustness of the overlapping area between the aortic wall and the stent-graft is a determining factor for the long-term durability of endovascular aortic repair (EVAR) [1, 2]. Stentgraft migration has a reported prevalence ranging from 1.1 to $28 \%$ [2-5]. It is responsible for the majority of late complications after EVAR, including late stent-graft-related endoleaks, resulting in aneurysm sac enlargement, and even rupture [6, 7]. Different mechanisms, such as the radial forces of selfexpandable stent-grafts due to oversizing $[8,9]$ and the pulsatile forces of blood flow [10-12], were suggested to be associated with continuous changes in stent-graft position and decreasing apposition of the stent-graft surface, consequentially causing migration over time. In addition, disease progression could trigger and accelerate both mechanisms [13, 14]. Stentgraft dynamics over time are complex and three-dimensional [15-17], and not only limited to a one-dimensional, most commonly caudal displacement of the stent-graft, as defined for migration [18]. Longitudinal displacement may occur simultaneously or consecutively with stent-graft surface movement in other directions $[16,19]$. Focusing only on caudal migration, stent-graft surface movement in other directions, as a result of subtle aneurysm neck changes, may be overlooked. Diagnosis of these subtle changes on regular computed tomography angiography (CTA) images is difficult, even with centerline reconstructions on a vascular workstation $[16,19]$. On the contrary, the calculation of three-dimensional (3D) surface models, derived from CTA images, enables a simplified visualization and assessment of stent-graft surface movement $[15,19]$, with the potential of a more sensitive determination of changes of the stent-graft position within the infrarenal aortic neck. Therefore, knowledge of the predisposing factors associated with the occurrence of stent-graft surface movement may influence the pre-operative planning in terms of patient selection for EVAR, the possible use of adjunctive sealing systems, and surveillance strategy. Detection of stent-graft surface movement during CTA surveillance, particularly in patients at risk, may allow for planning of timely prophylactic re-interventions to prevent stent-graft migration and consecutive sealing loss.

The primary objective of this study was to evaluate the influence of baseline clinical and morphological parameters on the probability of stent-graft surface movement in patients undergoing EVAR. The second aim was to investigate the association of stent-graft surface movement with migration and stent-graft-related endoleaks (type 1 and 3), representing the leading causes of rupture after EVAR [20, 21].

\section{Materials and methods}

\section{Study design}

This was a retrospective single-center, cross-sectional study of patients with abdominal aortic aneurysm (AAA) who were followed after elective EVAR. The institutional review board (No. 1843/2018) approved the study protocol and waived written, informed consent.

\section{Study population}

Our institutional database was screened for patients who underwent elective endovascular AAA repair between May 2002 and July 2014 and who met the following inclusion criteria: (1) EVAR with bifurcated stent-grafts without intraprocedure implantation of proximal adjunctive sealing devices and (2) available standardized CTA, (a) pre-procedure, within 3 months prior to intervention, (b) pre-discharge within 1 week after intervention, and (c) after a time interval of at least 6 months.

All patient data were anonymized and de-identified prior to analysis. One hundred eighty-seven patients (14 female) with a mean age of $73.2 \pm 8.0$ (range, 52-90) years were available for final analysis. Details of patient selection and patient characteristics are given in Fig. 1 and Table 1, respectively. A part of the study population was included in a previously described cohorts $[15,46]$. In contrast to our study, analyses referred on the comparison of stent-graft surface movement in patients with and without a type 2 endoleak, and clinical and morphological parameters for the prediction of late stent-graft related endoleaks, respectively.

\section{CTA examination protocol and stent-graft surface movement}

CTAs were performed in the caudo-cranial direction using a 16-slice (Somatom Sensation 16, Siemens Healthineers; $n=$ 


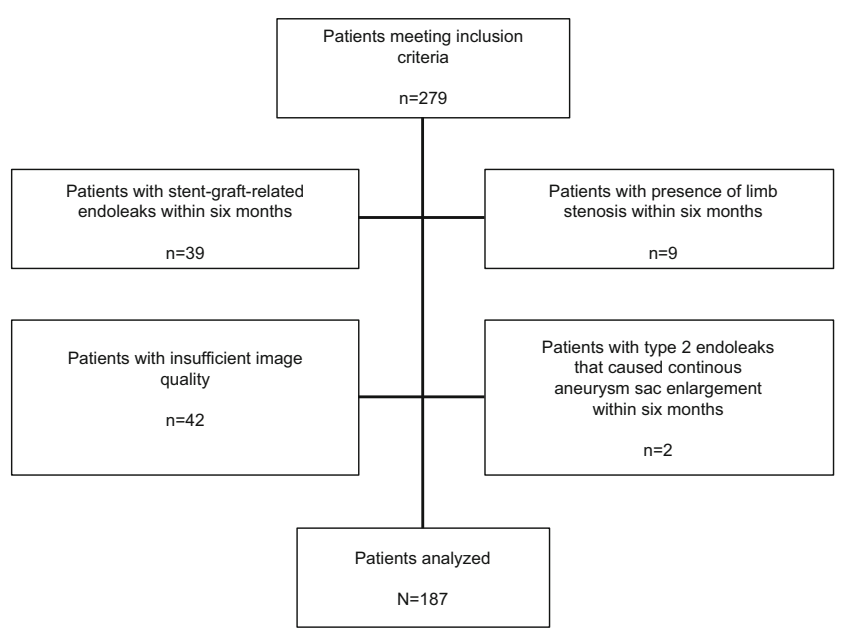

Fig. 1 Flow diagram showing patient selection

122) or a dual-source (Somatom Definition Flash; Siemens Healthineers; $n=65$ ) scanner. The institutional standard protocol included an arterial phase, ranging from the celiac trunk to the groin solely for pre-interventional imaging, followed by a late phase limited to the extent of the stent-graft in case of

Table 1 Patient characteristics

\begin{tabular}{lc}
\hline & $N(\%)$ \\
\hline Hypertension & $183(97.9 \%)$ \\
Hyperuricemia & $67(35.8 \%)$ \\
Hyperlipidemia & $139(74.3 \%)$ \\
History of stroke & $27(14.4 \%)$ \\
PAOD (Fontaine stage) & $35(18.7 \%)$ \\
Stage I & $2(1.1 \%)$ \\
Stage IIa & $7(3.7 \%)$ \\
Stage IIb & $18(9.6 \%)$ \\
Stage III & $3(1.6 \%)$ \\
Stage IV & $5(2.7 \%)$ \\
Atrial fibrillation & $27(14.4 \%)$ \\
Cardiac pacemaker & $9(4.8 \%)$ \\
Coronary heart disease & $92(49.2 \%)$ \\
MCI & $65(34.8 \%)$ \\
Diabetes mellitus & $32(17.1 \%)$ \\
IDDM & $5(2.7 \%)$ \\
NIDDM & $27(14.4 \%)$ \\
Renal insufficiency, mild to moderate & $58(30.0 \%)$ \\
Hemodialysis & $2(1.1 \%)$ \\
Smoking & $79(42.2 \%)$ \\
History of cancer & $43(23.0 \%)$ \\
\hline
\end{tabular}

$P A O D$ peripheral artery occlusive disease, $M C I$ myocardial infarction, IDDM insulin-dependent diabetes mellitus, NIDDM noninsulindependent diabetes mellitus postinterventional imaging. Acquisition parameters are given in Table 2.

Stent-graft surface movement was assessed as described by Nolz et al [15]. In short, three-dimensional (3D) surface models of the stent-graft, delimited by landmarks using custom-built software, were derived from arterial CTA images. The software calculated a vector for each point of the stent-graft surface, describing the surface movement between the postprocedure and final follow-up CTA, which was given in millimeters. Based on results of previous studies [15, 22], where significantly higher rates of EVAR failure were reported in patients with a stent-graft movement more than $9 \mathrm{~mm}$, this threshold was considered significant.

\section{Measurements and definitions}

The influence of baseline clinical and morphological parameters on the probability of stent-graft surface movement $>9 \mathrm{~mm}$ in the proximal anchoring zone was analyzed. As previously reported [15], this zone was defined as the proximal $3 \mathrm{~cm}$ of the covered stent-graft.

Baseline clinical parameters included age (at the time of EVAR), sex, and body mass index (BMI). Baseline morphological parameters included anatomical variables measured in the pre-procedural CTA scans, device-dependent variables collected from the pre-interventional CTA and/or the stentgraft procedure, and early postinterventional variables assessed on the pre-discharge CTA. Measurements were performed with the syngo.via imaging software (Siemens Healthineers).

Anatomical variables determined in accordance with the Society for Vascular Surgery standards for EVAR [23] included (1) aneurysm sac diameter, (2) maximum diameter of the proximal anchoring zone, (3) iliac sealing diameters, and (4) proximal and distal neck lengths. The supra- and infrarenal aortic neck angulations were measured based on the 2D methodology described by van Keulen et al [24], and adapted for measuring along a semi-automatically drawn center lumen line (CLL). Assessment of the psoas muscle area (PMA) was performed as described by Indrakusuma et al [25]. The area of both psoas muscles was added and corrected for patient height using the formula (left PMA + right PMA)/ $\left(\right.$ height $\left.^{2}\right)$ [26]. This variable was determined as heightcorrected PMA.

The presence of a thrombus with $>2 \mathrm{~mm}$ thickness at the circumference of the proximal anchoring zone was evaluated and classified as follows: (1) no thrombus, (2) thrombus $<25 \%$, (3) thrombus $25-50 \%$, or (4) thrombus $\geq 50 \%$. The presence of calcifications was categorized according to the same classification. On the basis of gathered anatomical variables, compliance requirements with the manufacturer's instructions for use (IFU) were analyzed, and rated as within or outside the IFU. 
Table 2 Acquisition parameters

\begin{tabular}{lll}
\hline & 16-slice scanner & Dual-source scanner \\
\hline Contrast media, ml & Biphasic: 30 and 85 & 110 \\
Injection rate, ml/s & Biphasic: 6 and 4.5 & 6 \\
Arterial phase & 6 s after threshold of $110 \mathrm{HU} *$ & $15 \mathrm{~s}$ after threshold of $150 \mathrm{HU}^{*}$ \\
Venous phase & $16 \mathrm{~s}$ after arterial phase & $18 \mathrm{~s}$ after arterial phase \\
Tube voltage $(\mathrm{kV})$ & 120 & Ref $120($ Care $\mathrm{kV})$ \\
Tube current (refmAs, CD4D) & 120 & 120 \\
Detector size/collimation $(\mathrm{mm})$ & $16 \times 0.75$ & $2 \times 64 \times 0.6$ \\
Rotation time (s) & 0.5 & 0.28 \\
Pitch & $\approx 1$ & $\approx 1$ \\
Soft kernel & B30 & $\mathrm{B} 30$ \\
Slice thickness/increment $(\mathrm{mm})$ & $1 / 0.8$ & $1 / 0.8$ \\
FOV & $\approx 300$ & $\approx 300$ \\
Spatial resolution, $x / y$ plane & $2 \%$ MTF $14.7 \mathrm{lp} / \mathrm{cm}( \pm 10 \%)$ & $2 \% \mathrm{MTF} 16.4 \mathrm{lp} / \mathrm{cm}( \pm 10 \%)$ \\
Spatial resolution, $z$ plane & $2 \%$ MTF $14.7 \mathrm{lp} / \mathrm{cm}( \pm 10 \%)$ & $2 \% \mathrm{MTF} 18.5 \mathrm{lp} / \mathrm{cm}( \pm 10 \%)$ \\
\hline
\end{tabular}

$\mathrm{ml}$ milliliters, $s$ seconds, $H U$ Hounsfield units, $k V$ kilovolt, $r e f m A s$ reference milliampereseconds, $m m$ millimeters, FOV field of view, MTF modulation transfer function, $l \mathrm{p} / \mathrm{cm}$ line pairs per centimeter

*Region of interest positioned in the aorta at the level of the celiac trunk (bolus tracking technique)

The followed devices were implanted: Talent (Medtronic; $n=36,19.3 \%$ ), Excluder (W.L. Gore \& Associates; $n=74$, $39.6 \%$ ), Zenith (Cook Medical; $n=20,10.7 \%$ ), Endurant (Medtronic; $n=46,24.6 \%$ ), Anaconda (Vascutek; $n=4$, $2.1 \%$ ), Aorfix (Lombard Medical Technologies; $n=1$, $0.5 \%$ ), Powerlink (Endologix, Inc.; $n=1,0.5 \%$ ), and Treovance (Bolton Medical; $n=5,2.7 \%$ ). Device-dependent variables included (1) proximal oversizing factor, (2) distal oversizing factors, (3) proximal fixation level (suprarenal/ infrarenal), (4) implantation side of the modular limb (right/ left), and (5) presence of any active fixation mechanism (e.g., hooks, anchoring pins, or dull barbs).

Early postinterventional variables included (1) the presence of a type 2 endoleak (T2EL) and (2) the renal artery to stent-graft distance (RSD). RSD was measured along a semi-automatically drawn CLL, as described by Bastos Goncalves et al [27]. RSD was defined as the distance between the lowest renal artery to the lowest stent-graft fabric marker, representing the level of circumferential stent-graft covering of the aortic wall. RSD changes between both examinations indicate stentgraft migration, which was defined as an RSD increase of $>0.5 \mathrm{~cm}$ [28]. Routinely performed CTA follow-up examinations were retrospectively screened for the presence of a stent-graft-related endoleak, type 1 or type 3 , as described by Chaikof et al [18].

\section{Statistical analysis}

Normally distributed, continuous data were presented as the mean \pm standard deviation. Potential differences between groups were compared using the $t$ test. Non-normally distributed data were described by medians and interquartile ranges (IQRs). Possible differences between groups were tested with the Wilcoxon-Mann-Whitney $U$ test. Dichotomous variables were described in absolute numbers and percentages, and possible differences between groups were tested by the chi-square test or the Fisher exact test, as appropriate. The univariate Cox proportional hazards model, with calculation of hazard ratios with $95 \%$ confidence intervals, was used to investigate the influence of baseline clinical and morphological parameters on the probability of stent-graft surface movement-free survival. A multivariate Cox proportional hazards model was conducted by using a backward selection of parameters, with a limit of $p<0.1$ required to enter and to stay in the model. The proportional hazards assumption was tested for each variable individually using the time-dependent covariate method [29]. No relevant violations of the assumption were found (see supplementary material). The effect of adding interaction terms was assessed using the partial likelihood ratio test. No statistically significant interactions were determinable. Additionally, receiver operating characteristics (ROC) analysis was performed in variables, significantly associated with stent-graft surface movement in the multivariate analysis. Cutoff values were defined with the Youden $J$ statistics. The Kaplan-Meier life table method was used to determine freedom from stent-graft surface movement, migration, and stentgraft-related endoleaks. For these survival analyses, all observations were censored at the time of the patient's last CTA. All tests were two-sided; significance was assumed at $p<0.05$. All statistical analyses were performed using SPSS for Windows (version 24.0; IBM Corporation). 


\section{Results}

Stent-graft surface movement more than $9 \mathrm{~mm}$ was detected in 54 (28.9\%) of 187 patients during a mean MSCTA follow-up of $33.5 \pm 25.4$ (median and IQR) months. Overall, cumulative freedom from stent-graft surface movement rates after 1,3 , and 5 years were $94.6 \%, 78.8 \%$, and $55.3 \%$, respectively (Fig. 2). Stent-graft migration occurred significantly $(p<0.001)$ more frequently in patients with $(n=17,31.5 \%)$, compared to those without $(n=11,8.3 \%)$, stent-graft surface movement (Fig. 3a-d). Late srEL occurred significantly $(p=$ $0.022)$ more frequently in patients with $(n=5,9.3 \%)$, compared to those without $(n=2,1.5 \%)$, stent-graft surface movement (Fig. 4a-d). Cumulative freedom from stent-graft migration and stent-graft-related endoleak rates after 1, 3, and 5 years were $98.1 \%, 90.9 \%$, and $66.8 \%$, as well as $100 \%$, $98.5 \%$, and $89.6 \%$, respectively (Figs. 5 and 6 ).

There were four (1.7\%) type 1a endoleaks caused by stentgraft migration that resulted in rupture in two patients, 45 and 42 months after treatment. Successful redo-EVAR was performed by implantation of an aorto-mono-iliac device implanted in one patient, and a bifurcated endoprosthesis in the second patient. The two other type 1a endoleaks were treated by implantation of proximal extensions (Endurant, Medtronic). Furthermore, two patients with a left-sided modular limb suffered from type $1 \mathrm{~b}$ endoleaks (one left, one right), which were treated by extension of the iliac limb into the external iliac artery after coil occlusion of the internal iliac artery. One type 3 endoleak, caused by disconnection of the left modular limb, was over-stented. Baseline clinical and morphological parameters of the entire cohort, separated for patients with and without stent-graft surface movement, are given in Table 3. Factors with a significance level $<0.1$ in the univariate analysis associated with stent-graft surface movement are given in Table 4. Multivariate analysis revealed that age ([HR] 1.05, 95\% CI 1.01-1.08; $p=0.017)$, proximal neck diameter ([HR] 5.07, 95\% CI 1.94-13.23; $p<0.001$ ), and infrarenal aortic neck angulation ([HR] 1.02, 95\% CI 1.01$1.03 ; p=0.002$ ) were significantly associated with a higher incidence of stent-graft surface movement. Proximal neck length ([HR] $0.62,95 \%$ CI $0.49-0.80 ; p<0.001$ ) proved to be significantly associated with a decreased incidence of stentgraft surface movement in the multivariate analysis (Table 4).

For identification of patients with stent-graft surface movement, the optimal cut-off points for age, neck diameter, neck length, and infrarenal neck angulation were 72.3 years, $2.65 \mathrm{~cm}, 4.15 \mathrm{~cm}$, and $39.5^{\circ}$ with areas under the curve of 0.538 (95\% CI $0.449-0.628 ; p=0.410), 0.607$ (95\% CI $0.519-0.695 ; p=0.022), 0.633(95 \%$ CI $0.546-0.719 ; p=$ $0.004)$, and $0.530(0.435-0.625 ; p=0.526)$, respectively.

\section{Discussion}

The present study analyzed the influence of baseline clinical and morphological parameters on the occurrence of stent-graft surface movement after endovascular aneurysm repair. A multivariate Cox model revealed a relation between stent-graft surface movement and patient age, the proximal aneurysm neck diameter as well as the infrarenal aortic neck angulation, whereas proximal neck length proved to be significantly
Fig. 2 Kaplan-Meier survival curve demonstrating freedom from stent-graft surface movement

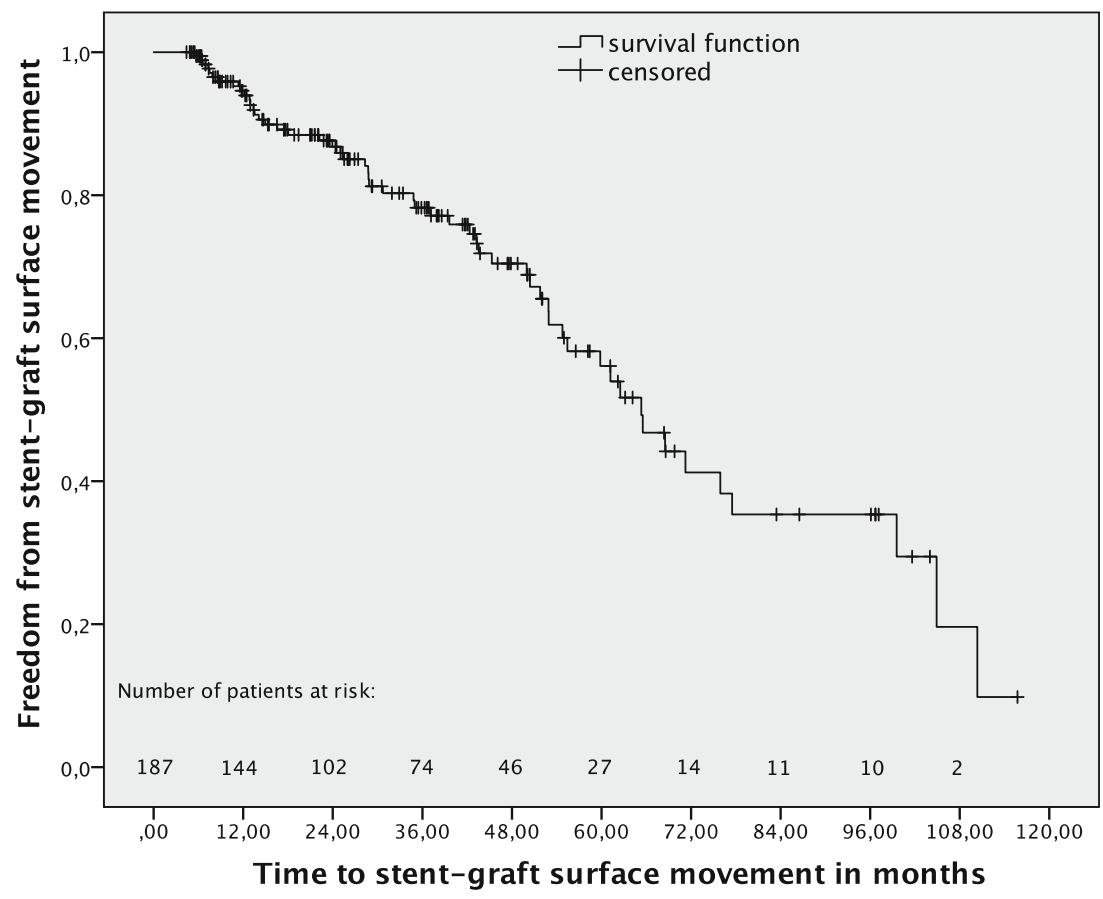




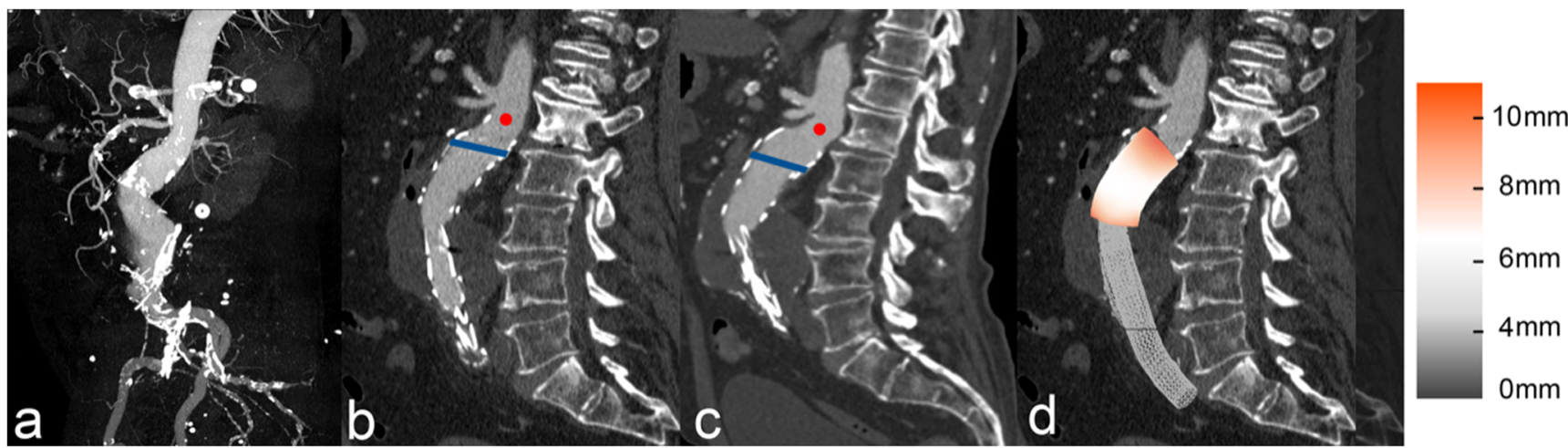

Fig. 3 a-d 92-year-old male patient with severe angulation and large diameter $(28 \mathrm{~mm})$ of the infrarenal neck on pre-procedural CTA-maximum intensity-projection images (a). Comparing sagittal pre-discharge (b) and 3-year follow-up (c) CTA images, widening of the proximal aortic neck diameter and significant migration (RSD $13 \mathrm{~mm}$ vs $25 \mathrm{~mm}$ ) were

associated with a decreased incidence of stent-graft surface movement.

Furthermore, stent-graft migration and srEL occurred significantly more frequently in patients with stent-graft surface movement.

EVAR should not be considered to be a single-step procedure, based on preoperative anatomical features alone. During pre-procedure planning, one should be aware of the ongoing process of conformational geometrical changes of the stentgraft and the diseased aorta $[30,31]$. In this context, an intact proximal sealing zone is of crucial importance for stent-graft stability and integrity, preventing distal migration, stent-graftrelated endoleaks, and, ultimately, aortic rupture. However, detection of these subtle changes on regular CTA images is difficult, even with centerline reconstructions on a vascular workstation $[16,19]$. A recent study by Schuurmann et al [19], using a semiautomatic software, observed significant changes in proximal stent-graft dimension and apposition on regular CTA scans prior to failure, when patients with and observed. The red dot $(\mathbf{b}, \mathbf{c})$ marks the lowest renal artery, while the blue line represents fabric markers of the stent-graft coverage. Significant stent-graft surface movement $(>9 \mathrm{~mm}$ ) occurred at the proximal landing zone, visualized by 3D matched surface models of postprocedural and final follow-up CTA (d)

without later stent-graft migration were compared. This observation was reflected in our results, where subtle changes of the stent-graft surface could be detected in $28.9 \%$ of patients using a custom-made semi-automatic software. Consistently, migration and srEL were significantly more frequent in patients with stent-graft surface movement.

The incidence of stent-graft migration in the present study was $15.0 \%$, which is in line with percentages $(1.1-28 \%)$ previously reported [2-5]. Migration bears the risk of sealing loss followed by late stent-graft-related endoleaks, which were observed in $3.7 \%$ of our patients. This incidence is lower compared with the percentages (3.9-10.6\%) previously reported [32-34], which may be explained by the exclusion of patients with early srEL and re-intervention within 6 months.

Different anatomical aspects of proximal neck morphology were found to be associated with the incidence of migration. In this context, the aortic neck diameter was found to be a risk factor for stent-graft migration [3, 4, 35], which is in accordance with our results where an increasing neck diameter was

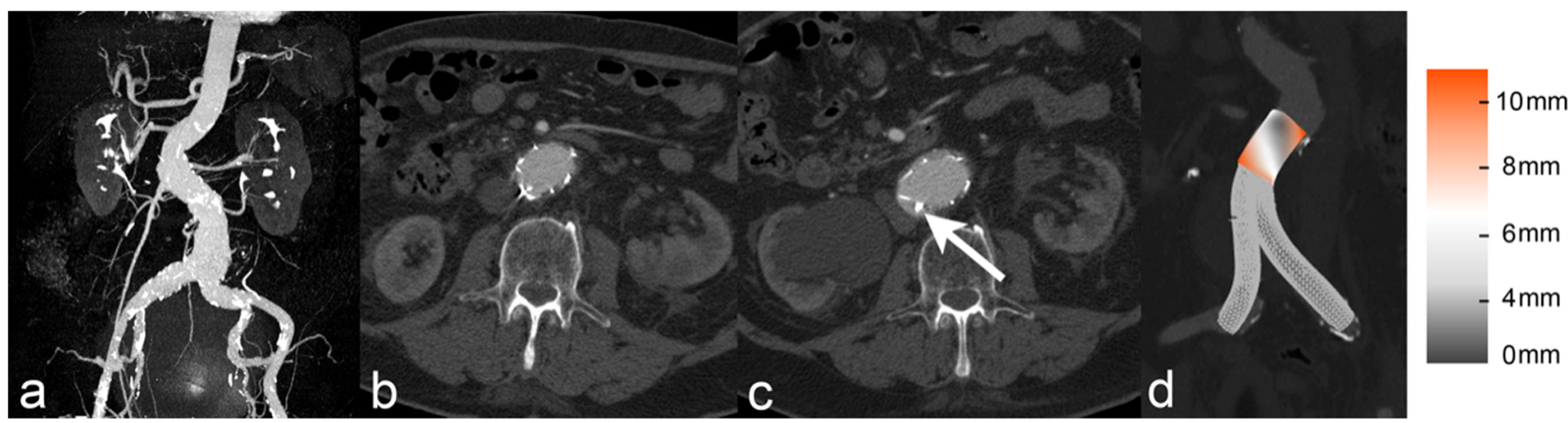

Fig. 4 a-d 83-year-old male patient with evident angulation and wide diameter of the infrarenal neck on pre-procedural CTA-maximum intensity-projection images (a). In comparison to the pre-discharge CTA (b), there is an obvious lateralization of the superior mesenteric artery and the abdominal aorta with consecutive compression of the inferior vena cava after 4 years of follow-up (c). Further, the aortic neck diameter increased from 29 to $36 \mathrm{~mm}$ with consecutive sealing loss at the dorsal circumference of the proximal anchoring zone and occurrence of a type 1a endoleak (arrow). Significant stent-graft surface movement ( $>9 \mathrm{~mm}$ ) at the proximal anchoring zone is visualized on $3 \mathrm{D}$ matched surface models of postprocedural and final follow-up CT (d) 
Fig. 5 Kaplan-Meier survival curve demonstrating freedom from stent-graft migration

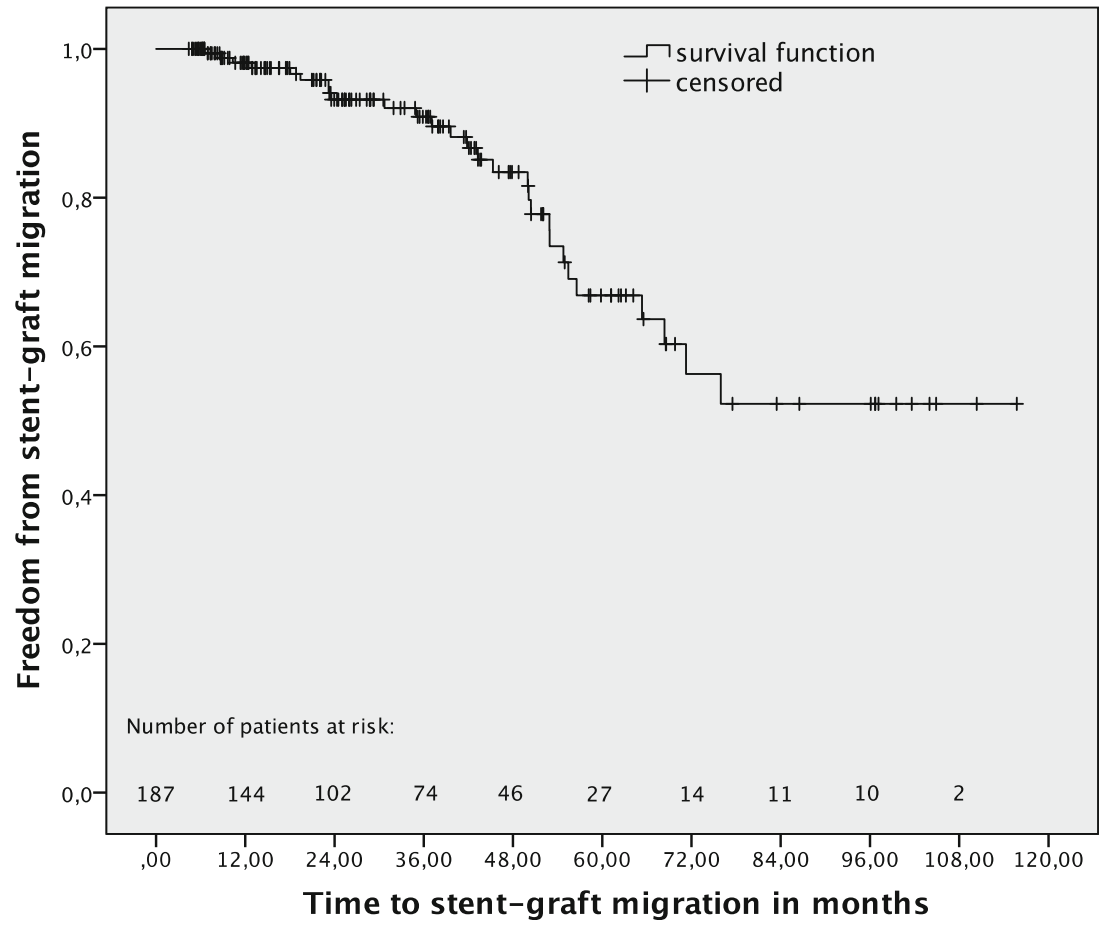

related to stent-graft surface movement in the multivariate analysis. Shorter neck length is a well-known risk factor for migration [36, 37], which is in line with our results, demonstrating the protective effect of longer neck lengths in terms of stent-graft surface movement. Another feature of hostile neck anatomy is angulation, which decreases the necessary pulldown force causally responsible for dislodgement of the stent-graft [38]. Consequently, neck angulation was found to be a risk factor for early migration [32] and type 1 endoleaks [39]. This mechanism is supported by our results, where neck angulation was found to be significantly associated with stentgraft surface movement in the multivariate analysis. Displacement forces and stent-graft stability are also related to the iliac fixation $[14,37]$. Through distal fixation, different authors have identified the docking area of the modular stent graft limb as a vulnerable region, which seems to be exposed
Fig. 6 Kaplan-Meier survival curve demonstrating freedom from stent-graft-related endoleak

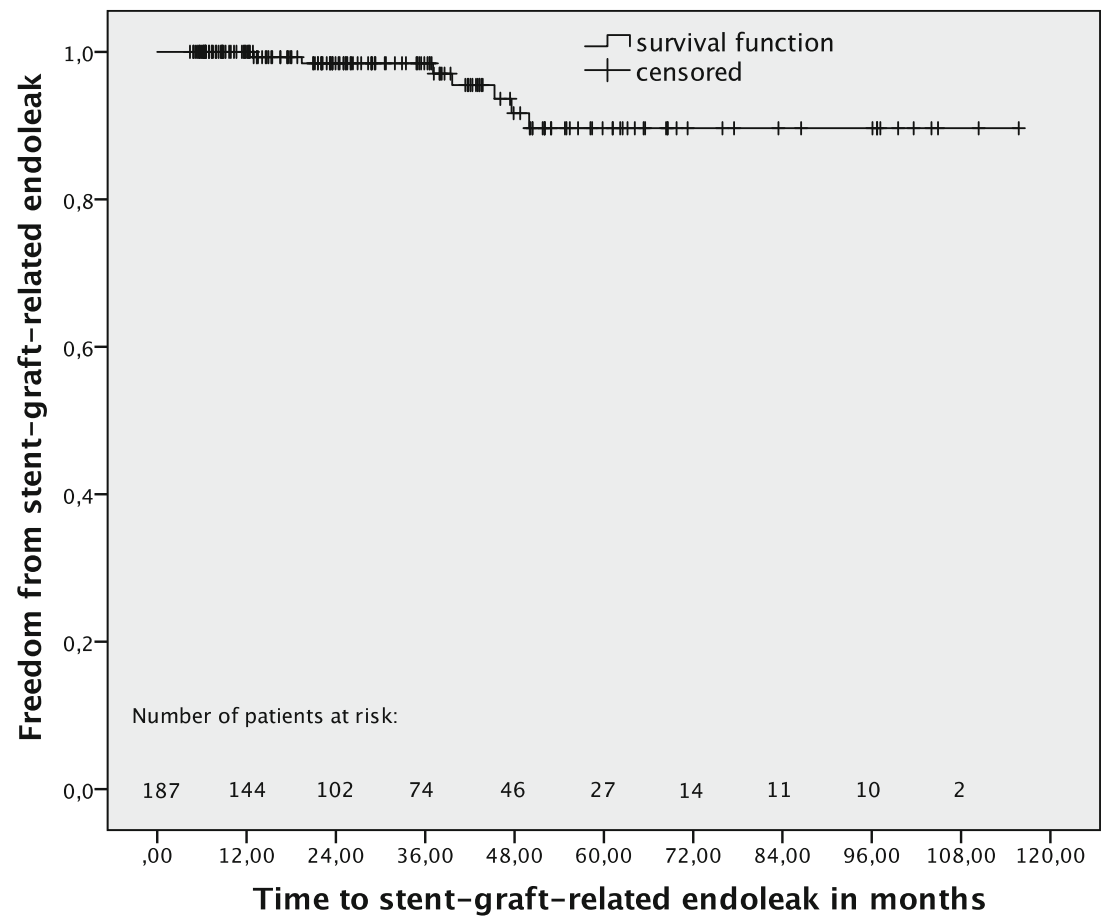


Table 3 Baseline clinical and morphological parameters

Overall $(n=187)$

Stent-graft surface movement

$(n=54)$
No stent-graft surface movement $\quad p$

$(n=133)$

\begin{tabular}{|c|c|c|c|c|}
\hline \multicolumn{5}{|l|}{ Baseline clinical variables } \\
\hline Sex, female & $14(7.5 \%)$ & $5(9.3 \%)$ & $9(6.8 \%)$ & 0.550 \\
\hline Age, years & $73.22 \pm 7.97$ & $74.19 \pm 7.72$ & $72.83 \pm 8.06$ & 0.288 \\
\hline Body mass index, $\mathrm{kg} / \mathrm{m}^{2}$ & $28.18 \pm 4.87$ & $28.62 \pm 5.57$ & $28.00 \pm 4.56$ & 0.427 \\
\hline \multicolumn{5}{|l|}{ Baseline anatomical variables } \\
\hline Aneurysm sac diameter, $\mathrm{cm}$ & $5.99 \pm 0.90$ & $6.07 \pm 0.97$ & $5.96 \pm 0.87$ & 0.451 \\
\hline Proximal neck length, $\mathrm{cm}$ & $3.08 \pm 1.28$ & $2.63 \pm 1.15$ & $3.26 \pm 1.30$ & $0.002 *$ \\
\hline Proximal neck diameter, $\mathrm{cm}$ & $2.42 \pm 0.31$ & $2.50 \pm 0.29$ & $2.39 \pm 0.31$ & $0.029 *$ \\
\hline Right iliac sealing diameter, $\mathrm{cm}$ & $1.25 \pm 0.22$ & $1.24 \pm 0.22$ & $1.25 \pm 0.22$ & 0.818 \\
\hline Right iliac neck length, $\mathrm{cm}$ & $1.72 \pm 0.56$ & $1.72 \pm 0.52$ & $1.72 \pm 0.58$ & 0.961 \\
\hline Left iliac sealing diameter, $\mathrm{cm}$ & $1.21 \pm 0.23$ & $1.24 \pm 0.26$ & $1.20 \pm 0.22$ & 0.261 \\
\hline Left iliac neck length, $\mathrm{cm}$ & $1.85 \pm 0.55$ & $1.85 \pm 0.61$ & $1.85 \pm 0.52$ & 0.997 \\
\hline Suprarenal aortic neck angulation, ${ }^{\circ}$ & $31.53 \pm 21.57$ & $29.85 \pm 21.18$ & $32.22 \pm 21.76$ & 0.498 \\
\hline Infrarenal aortic neck angulation, ${ }^{\circ}$ & $34.67 \pm 23.52$ & $36.93 \pm 26.11$ & $33.76 \pm 22.24$ & 0.406 \\
\hline Height-corrected PMA, $\mathrm{cm}^{2} / \mathrm{m}^{2}$ & $5.52 \pm 1.47$ & $5.52 \pm 1.62$ & $5.51 \pm 1.41$ & 0.968 \\
\hline $\begin{array}{l}\text { No neck thrombus } \\
<25 \%\end{array}$ & $\begin{array}{l}80(42.8 \%) \\
32(17.1 \%)\end{array}$ & $\begin{array}{l}15(27.8 \%) \\
12(22.2 \%)\end{array}$ & $\begin{array}{l}65(48.9 \%) \\
20(15.0 \%)\end{array}$ & $0.008 *$ \\
\hline $25-50 \%$ & $44(23.5 \%)$ & $18(33.3 \%)$ & $26(19.5 \%)$ & \\
\hline$\geq 50 \%$ & $31(16.6 \%)$ & $9(16.7 \%)$ & $22(16.5 \%)$ & \\
\hline $\begin{array}{l}\text { No neck calcification } \\
<25 \%\end{array}$ & $\begin{array}{r}163(87.2 \%) \\
19(10.2 \%)\end{array}$ & $\begin{array}{r}47(87.0 \%) \\
6(11.1 \%)\end{array}$ & $\begin{array}{c}116(87.2 \%) \\
13(9.8 \%)\end{array}$ & 0.973 \\
\hline $25-50 \%$ & $4(2.1 \%)$ & $1(1.9 \%)$ & $3(2.3 \%)$ & \\
\hline$\geq 50 \%$ & $1(0.5 \%)$ & 0 & $1(0.8 \%)$ & \\
\hline IFU, outside & $39(20.9 \%)$ & $16(29.6 \%)$ & $23(17.3 \%)$ & 0.060 \\
\hline \multicolumn{5}{|l|}{ Device-dependent variables } \\
\hline Proximal oversizing, $\%$ & $16.93 \pm 6.94$ & $16.46 \pm 6.50$ & $17.12 \pm 7.12$ & 0.556 \\
\hline Right distal oversizing, $\%$ & $17.00 \pm 7.82$ & $18.29 \pm 9.41$ & $16.47 \pm 7.04$ & 0.150 \\
\hline Left distal oversizing, $\%$ & $17.94 \pm 9.64$ & $17.04 \pm 8.20$ & $18.30 \pm 10.18$ & 0.421 \\
\hline $\begin{array}{l}\text { Fixation level, suprarenal } \\
\text { Fixation level, infrarenal }\end{array}$ & $\begin{array}{r}107(57.2 \%) \\
80(42.8 \%)\end{array}$ & $\begin{array}{l}38(70.4 \%) \\
16(29.6 \%)\end{array}$ & $\begin{array}{l}69(51.9 \%) \\
64(56.6 \%)\end{array}$ & $0.021 *$ \\
\hline $\begin{array}{l}\text { Active fixation mechanism } \\
\text { No active fixation mechanism }\end{array}$ & $\begin{array}{r}150(80.2 \%) \\
37(19.8 \%)\end{array}$ & $\begin{array}{l}37(68.5 \%) \\
17(31.5 \%)\end{array}$ & $\begin{array}{r}113(85.0 \%) \\
20(15.0 \%)\end{array}$ & $0.011^{*}$ \\
\hline Implantation side of modular limb, left & $146(78.1 \%)$ & $40(74.1 \%)$ & $106(79.7 \%)$ & 0.399 \\
\hline \multicolumn{5}{|l|}{ Early postinterventional variables } \\
\hline Type 2 endoleak & $94(50.3 \%)$ & $21(38.9 \%)$ & $73(54.9 \%)$ & $0.047 *$ \\
\hline Lumbar & $53(28.3 \%)$ & $10(18.5 \%)$ & $43(32.3 \%)$ & \\
\hline Lumbar + IMA & $20(10.7 \%)$ & $4(7.4 \%)$ & $16(12.0 \%)$ & \\
\hline IMA & $20(10.7 \%)$ & $7(13.0 \%)$ & $13(9.8 \%)$ & \\
\hline Accessory renal artery & $1(0.5 \%)$ & 0 & $1(0.8 \%)$ & \\
\hline $\mathrm{RSD}, \mathrm{mm}$ & 0 (IQR 0-0.40) & 0 (IQR 0-0.43) & 0 (IQR 0-0.40) & 0.575 \\
\hline
\end{tabular}

Comparisons between groups were performed with the $t$ test, the Wilcoxon-Mann-Whitney $U$ test, the chi-square test, or the Fisher exact test, as appropriate

PMA psoas muscle area, IMA internal mesenteric artery, RSD renal atery to stent-graft distance, $p$ patients with versus without stent-graft surface movement

*Indicates a significant difference

to the highest mechanical stress [31] and surface movement [15]. Taking into account that the modular limb was predominantly left-sided (78\%) in our cohort, the left iliac sealing diameter was significantly associated with an increased stent-graft surface movement in the univariate, but not in the multivariate, analysis. 
Table 4 Results of Cox regression analysis to identify stent-graft surface movement baseline factors associated with

\begin{tabular}{llllr}
\hline $\begin{array}{l}N=187 \\
\text { Co-variable }\end{array}$ & $\begin{array}{l}\text { Univariate } \\
\text { Hazard ratio }\end{array}$ & $\begin{array}{l}\text { Wald test } \\
p\end{array}$ & $\begin{array}{l}\text { Multivariate } \\
\text { Hazard ratio }\end{array}$ & $\begin{array}{l}\text { Wald test } \\
p\end{array}$ \\
\hline Age, years & $1.04(1.01-1.08)$ & $0.023^{*}$ & $1.05(1.01-1.08)$ & $0.017^{*}$ \\
Aneurysm sac diameter, cm & $1.32(0.96-1.80)$ & 0.085 & & \\
Proximal neck length, cm & $0.68(0.53-0.87)$ & $0.002^{*}$ & $0.62(0.49-0.80)$ & $<0.001^{*}$ \\
Proximal neck diameter, cm $_{\text {Infrarenal aortic neck angulation, }}{ }^{\circ}$ & $4.04(1.66-9.86)$ & $0.002^{*}$ & $5.07(1.94-13.23)$ & $0.001^{*}$ \\
Proximal fixation level suprarenal $^{*}$ & $2.68(1.46-4.90)$ & $0.001^{*}$ & & $0.002^{*}$ \\
Proximal oversizing, $\%$ & $0.96(0.93-1.00)$ & 0.065 & & \\
IFU, outside & $1.96(1.08-3.54)$ & $0.027^{*}$ & & \\
Left iliac diameter, cm & $2.82(0.95-8.38)$ & 0.062 & & \\
\hline
\end{tabular}

IFU instructions for use

*Indicates a significant difference

Another mechanism with a negative influence on wall compliance and aneurysm morphology is atherosclerotic disease progression [40], which particularly affects elderly patients [41]. Several authors reported an association between early stent-graft complications, including migration [42, 43], and increasing age. These findings were supported by our data, where increasing age was significantly associated with stent-graft surface movement in the multivariate analysis.

Different approaches were made to streamline the surveillance protocol proposed by the Society of Vascular Surgery [18], designed to reduce radiation exposure and the total costs of EVAR. As outlined by Hoel and Schanzer [44, 45], there should be a balance between adequate disease detection and unnecessary use of resources in postoperative surveillance. Knowledge of the predisposing factors associated with the occurrence of stent-graft surface movement may help to identify patients who benefit from regular CTA surveillance. Detection of subtle changes of the stent-graft position in these patients may allow for planning of timely prophylactic reinterventions to prevent stent-graft migration and consecutive sealing loss [19].

\section{Limitations}

Our study has several limitations. First, data were evaluated in a retrospective manner and we had to exclude 50 patients with early re-interventions for stent-graft failure, rendering the assessment of stent-graft surface movement impossible. Since we have no information on possible late complications of these patients, we cannot completely rule out a possible selection bias. Second, inclusion of second-generation devices and rudimentary planning in the early period of our study may have negatively affected the outcome in certain patients. Third, comparing the pre-discharge and the last CTA followup resulted in a heterogeneous time interval for measurements. As a consequence, the study design does not allow a conclusion about the time point for the occurrence of stent-graft surface movement and migration, nor on its chronological sequence. Fourth, due to the relatively small sample size and heterogeneity by means of implanted devices, we are not able to draw a reasonable statement on the performance of different devices.

\section{Conclusion}

Older patients, those with larger aneurysm neck diameters, and those with increased infrarenal aortic neck angulation experienced higher rates of stent-graft surface movement. Consideration of these risk factors may help to identify patients who benefit from possible use of adjunctive, prophylactic sealing systems and a regular CTA surveillance or even open repair as a primary intervention. Detection of stentgraft surface movement during CTA surveillance may allow for planning of timely prophylactic re-interventions to prevent stent-graft migration and stent-graft-related endoleaks.

Funding Information Open access funding provided by Medical University of Vienna.

\section{Compliance with ethical standards}

Guarantor The scientific guarantor of this publication is Richard Nolz.

Conflict of interest The authors declare that they have no competing interests.

Statistics and biometry No complex statistical methods were necessary for this paper.

As suggested by the reviewers, an expert in statistics was consulted for revision of the statistics section.

Pascal Baltzer, MD

Medical University of Vienna, Austria, Department of Bio-medical Imaging and Image-guided Therapy, Division of Cardiovascular and Interventional Radiology.

Pascal Baltzer, MD was added as one of the authors. 
Informed consent Written informed consent was waived by the Institutional Review Board.

Ethical approval Institutional Review Board approval was obtained.

Study subjects or cohorts overlap Some study subjects or cohorts have been previously reported in the European Journal of Vascular and Endovascular Surgery by Nolz et al [15, 46].

\section{Methodology \\ - retrospective \\ - cross-sectional study \\ - performed at one institution}

This retrospective observational study followed the checklist of items as published in the STROBE guidelines.

Open Access This article is distributed under the terms of the Creative Commons Attribution 4.0 International License (http:// creativecommons.org/licenses/by/4.0/), which permits unrestricted use, distribution, and reproduction in any medium, provided you give appropriate credit to the original author(s) and the source, provide a link to the Creative Commons license, and indicate if changes were made.

\section{References}

1. Prasad A, Xiao N, Gong XY, Zarins CK, Figueroa CA (2013) A computational framework for investigating the positional stability of aortic endografts. Biomech Model Mechanobiol 12:869-887

2. Zarins CK, Bloch DA, Crabtree T, Matsumoto AH, White RA, Fogarty TJ (2003) Stent graft migration after endovascular aneurysm repair: importance of proximal fixation. J Vasc Surg 38:1264 1272

3. Cao P, Verzini F, Zannetti S et al (2002) Device migration after endoluminal abdominal aortic aneurysm repair: analysis of 113 cases with a minimum follow-up period of 2 years. J Vasc Surg $35: 229-235$

4. van Herwaarden JA, van de Pavoordt ED, Waasdorp EJ et al (2007) Long-term single-center results with AneuRx endografts for endovascular abdominal aortic aneurysm repair. J Endovasc Ther 14:307-317

5. Spanos K, Karathanos C, Saleptsis V, Giannoukas AD (2016) Systematic review and meta-analysis of migration after endovascular abdominal aortic aneurysm repair. Vascular 24:323-336

6. De Bruin JL, Baas AF, Buth J et al (2010) Long-term outcome of open or endovascular repair of abdominal aortic aneurysm. N Engl J Med 362:1881-1889

7. The United Kingdom EVAR Trial Investigators, Greenhalgh RM, Brown LC et al (2010) Endovascular versus open repair of abdominal aortic aneurysm. N Engl J Med 362:1863-1871

8. Monahan TS, Chuter TA, Reilly LM, Rapp JH, Hiramoto JS (2010) Long-term follow-up of neck expansion after endovascular aortic aneurysm repair. J Vasc Surg 52:303-307

9. Savlovskis J, Krievins D, de Vries JP et al (2015) Aortic neck enlargement after endovascular aneurysm repair using balloonexpandable versus self-expanding endografts. J Vasc Surg 62: 541-549

10. Figueroa CA, Taylor CA, Yeh V, Chiou AJ, Gorrepati ML, Zarins CK (2010) Preliminary 3D computational analysis of the relationship between aortic displacement force and direction of endograft movement. J Vasc Surg 51:1488-1497
11. Molony DS, Kavanagh EG, Madhavan P, Walsh MT, McGloughlin TM (2010) A computational study of the magnitude and direction of migration forces in patient-specific abdominal aortic aneurysm stent-grafts. Eur J Vasc Endovasc Surg 40:332-339

12. van Keulen JW, Moll FL, Barwegen GK, Vonken EP, van Herwaarden JA (2010) Pulsatile distension of the proximal aneurysm neck is larger in patients with stent graft migration. Eur J Vasc Endovasc Surg 40:326-331

13. Pintoux D, Chaillou P, Azema L et al (2011) Long-term influence of suprarenal or infrarenal fixation on proximal neck dilatation and stentgraft migration after EVAR. Ann Vasc Surg 25:1012-1019

14. Georgakarakos E, Argyriou C, Schoretsanitis N et al (2014) Geometrical factors influencing the hemodynamic behavior of the AAA stent grafts: essentials for the clinician. Cardiovasc Intervent Radiol 37:1420-1429

15. Nolz R, Schwartz E, Langs G et al (2015) Stent graft surface movement after infrarenal abdominal aortic aneurysm repair: comparison of patients with and without a type 2 endoleak. Eur J Vasc Endovasc Surg 50:181-188

16. Waasdorp EJ, Gorrepati ML, Rafii BY, de Vries JP, Zarins CK (2012) Sideways displacement of the endograft within the aneurysm sac is associated with late adverse events after endovascular aneurysm repair. J Vasc Surg 55:947-955

17. van Keulen JW, Vincken KL, van Prehn J et al (2010) The influence of different types of stent grafts on aneurysm neck dynamics after endovascular aneurysm repair. Eur J Vasc Endovasc Surg 39:193-199

18. Chaikof EL, Dalman RL, Eskandari MK et al (2018) The Society for Vascular Surgery practice guidelines on the care of patients with an abdominal aortic aneurysm. J Vasc Surg 67:2-77

19. Schuurmann RCL, van Noort K, Overeem SP et al (2018) Determination of endograft apposition, position, and expansion in the aortic neck predicts type Ia endoleak and migration after endovascular aneurysm repair. J Endovasc Ther 25:366-375

20. Schlosser FJ, Gusberg RJ, Dardik A et al (2009) Aneurysm rupture after EVAR: can the ultimate failure be predicted? Eur J Vasc Endovasc Surg 37:15-22

21. Antoniou GA, Georgiadis GS, Antoniou SA et al (2015) Late rupture of abdominal aortic aneurysm after previous endovascular repair: a systematic review and meta-analysis. J Endovasc Ther 22: 734-744

22. Rafii BY, Abilez OJ, Benharash P, Zarins CK (2008) Lateral movement of endografts within the aneurysm sac is an indicator of stentgraft instability. J Endovasc Ther 15:335-343

23. Chaikof EL, Blankensteijn JD, Harris PL et al (2002) Reporting standards for endovascular aortic aneurysm repair. J Vasc Surg 35:1048-1060

24. van Keulen JW, Moll FL, Tolenaar JL, Verhagen HJ, van Herwaarden JA (2010) Validation of a new standardized method to measure proximal aneurysm neck angulation. J Vasc Surg 51: $821-828$

25. Indrakusuma R, Zijlmans JL, Jalalzadeh H, Planken RN, Balm R, Koelemay MJW (2018) Psoas muscle area as a prognostic factor for survival in patients with an asymptomatic infrarenal abdominal aortic aneurysm: a retrospective cohort study. Eur J Vasc Endovasc Surg 55:83-91

26. Cruz-Jentoft AJ, Baeyens JP, Bauer JM et al (2010) Sarcopenia: European consensus on definition and diagnosis: report of the European Working Group on Sarcopenia in Older People. Age Ageing 39:412-423

27. Bastos Goncalves F, van de Luijtgaarden KM, Hoeks SE et al (2013) Adequate seal and no endoleak on the first postoperative computed tomography angiography as criteria for no additional imaging up to 5 years after endovascular aneurysm repair. J Vasc Surg 57:1503-1511 
28. Karthikesalingam A, Holt PJ, Vidal-Diez A et al (2013) Predicting aortic complications after endovascular aneurysm repair. Br J Surg 100:1302-1311

29. Ng'andu NH (1997) An empirical comparison of statistical tests for assessing the proportional hazards assumption of Cox's model. Stat Med 16:611-626

30. Georgakarakos E, Georgiadis GS, Ioannou CV, Kapoulas KC, Trellopoulos G, Lazarides M (2012) Aneurysm sac shrinkage after endovascular treatment of the aorta: beyond sac pressure and endoleaks. Vasc Med 17:168-173

31. Kramer SC, Seifarth H, Pamler R, Fleiter T, Gorich J (2001) Geometric changes in aortic endografts over a 2-year observation period. J Endovasc Ther 8:34-38

32. Hobo R, Kievit J, Leurs LJ, Buth J, Collaborators E (2007) Influence of severe infrarenal aortic neck angulation on complications at the proximal neck following endovascular AAA repair: a EUROSTAR study. J Endovasc Ther 14:1-11

33. Bastos Goncalves F, Baderkhan H, Verhagen HJ et al (2014) Early sac shrinkage predicts a low risk of late complications after endovascular aortic aneurysm repair. Br J Surg 101:802-810

34. Wyss TR, Dick F, Brown LC, Greenhalgh RM (2011) The influence of thrombus, calcification, angulation, and tortuosity of attachment sites on the time to the first graft-related complication after endovascular aneurysm repair. J Vasc Surg 54:965-971

35. Howard DPJ, Marron CD, Sideso E et al (2018) Editor's choice influence of proximal aortic neck diameter on durability of aneurysm sealing and overall survival in patients undergoing endovascular aneurysm repair. Real world data from the Gore Global Registry for Endovascular Aortic Treatment (GREAT). Eur J Vasc Endovasc Surg 56:189-199

36. Bastos Goncalves F, Hoeks SE, Teijink JA et al (2015) Risk factors for proximal neck complications after endovascular aneurysm repair using the endurant stentgraft. Eur J Vasc Endovasc Surg 49: $156-162$

37. Waasdorp EJ, de Vries JP, Sterkenburg A et al (2009) The association between iliac fixation and proximal stent-graft migration during EVAR follow-up: mid-term results of 154 Talent devices. Eur J Vasc Endovasc Surg 37:681-687
38. Rahmani S, Grewal IS, Nabovati A, Doyle MG, Roche-Nagle G, Tse LW (2016) Increasing angulation decreases measured aortic stent graft pullout forces. J Vasc Surg 63:493-499

39. Oliveira NFG, Goncalves FB, Hoeks SE et al (2018) Long-term outcomes of standard endovascular aneurysm repair in patients with severe neck angulation. J Vasc Surg 68:1725-1735

40. Ailawadi G, Eliason JL, Roelofs KJ et al (2004) Gender differences in experimental aortic aneurysm formation. Arterioscler Thromb Vasc Biol 24:2116-2122

41. Paneni F, Diaz Canestro C, Libby P, Luscher TF, Camici GG (2017) The aging cardiovascular system: understanding it at the cellular and clinical levels. J Am Coll Cardiol 69:1952-1967

42. Brown LC, Greenhalgh RM, Powell JT, Thompson SG, Participants ET (2010) Use of baseline factors to predict complications and reinterventions after endovascular repair of abdominal aortic aneurysm. Br J Surg 97:1207-1217

43. EVAR trial participants (2005) Endovascular aneurysm repair and outcome in patients unfit for open repair of abdominal aortic aneurysm (EVAR trial 2): randomised controlled trial. Lancet 365:21872192

44. Hoel AW, Schanzer A (2015) Follow-up surveillance after endovascular aneurysm repair: less is more? JAMA Surg 150:964

45. Schanzer A, Messina LM, Ghosh K et al (2015) Follow-up compliance after endovascular abdominal aortic aneurysm repair in Medicare beneficiaries. J Vasc Surg 61:16-22

46. Nolz R, Schoder M, Baltzer P, Prusa A, Javor D, Loewe D, Asenbaum U, (2019) Application of Baseline Clinical and Morphological Parameters for Prediction of Late Stent Graft Related Endoleaks after Endovascular Repair of Abdominal Aortic Aneurysm. European Journal of Vascular and Endovascular Surgery

Publisher's note Springer Nature remains neutral with regard to jurisdictional claims in published maps and institutional affiliations. 